\title{
Chikungunya in the region of the Americas. A challenge for rheumatologists and health care systems
}

\author{
Carlos Pineda $^{1} \cdot$ Roberto Muñoz-Louis $^{2} \cdot$ Carlo V. Caballero-Uribe $^{3} \cdot$ Diego Viasus $^{4}$
}

Received: 16 August 2016/Accepted: 17 August 2016/Published online: 24 August 2016

(C) International League of Associations for Rheumatology (ILAR) 2016

\begin{abstract}
At the end of 2013, the Pan American Health Organization issued an epidemiological alert due to the detection of the first local cases of Chikungunya in the Americas. By August 2015, autochthonous transmissions were detected in 33 countries and territories of the Americas. Latin America has reported nearly one million cases; only Colombia has issued a report of $>200,000$ cases during the first 4 months of 2015. In some Latin American and Caribbean countries, Chikungunya becomes a major public health problem. The disease commonly exhibits a self-limited course of arthritis, usually lasting for a few days or that may be prolonged to weeks; however, in 10-60\% of cases, joint pain may become chronic and persist for up to 3-5 years. Human-caused environmental changes, such as climate change, the globalization of international exchange, and disordered urban growth, are some factors that aid in its emergence and dissemination. Outbreaks of Chikungunya comprise a challenge for health care systems and rheumatologists because of the high attack rate on the population and the anticipated development of post-Chikungunya chronic rheumatism. This review emphasizes the rheumatologic clinical manifestations reported in the American continent and highlights the challenges that health care systems face in the absence of an effective vaccine and specific treatment to fight Chikungunya.
\end{abstract}

Roberto Muñoz-Louis

rmunozlouis@gmail.com

Instituto Nacional de Rehabilitación, Mexico City, Mexico

2 Hospital Docente Padre Billini, 10210 Santo Domingo, Dominican Republic

3 Universidad del Norte, Barranquilla, Colombia

4 Universidad del Norte and Hospital Universidad del Norte, Barranquilla, Colombia
Keywords Arbovirus · Arthritis · Chikungunya Epidemiology $\cdot$ Health care systems $\cdot$ Outbreak

Infectious diseases can be distinguished from other human diseases because of their unpredictable and explosive global character, their transmissibility, the close relationship between the environment and human behavior, and their ability to be prevented and eradicated [1]. In recent years, we have seen the emergence of some arboviral diseases transmitted by mosquitoes in different countries and territories of the Americas, in particular, Chikungunya (CHIK) [2].

CHIK (CIE-10A 92.0) is an emerging, arthritogenic disease caused by a single-stranded RNA arbovirus, belonging to the family Togaviridae and genus Alphavirus, CHIK virus (CHIKV). Aedes aegypti, and the more coldtolerant Aedes albopictus, mosquitoes, the same species involved in the transmission of dengue and Zika transmit the disease. The natural cycle of the virus is human-mosquito-human [3-5].

CHIKV was first isolated from the serum of a febrile patient during an outbreak that occurred in a southern province of Tanzania in 1952-1953 [6, 7]. The name "chikungunya" derives from "kungunyala" that, in the Kimakonde language, means "to become contorted" and that describes the stooped appearance of sufferers with joint pain. Its incapacitating arthralgias also account for its other name, "buka-buka," meaning "broken-broken," by which it is known in the Congo region [3].

Following the report from Tanganyika in 1952 [6, 7], CHIK epidemics have been reported from several parts of the world, including Asia, Africa, Oceania, and Europe [8-11]. This recent re-emergence of CHIKV has given rise to the growing concern and interest regarding the impact of this virus on global public health. 
On December 9, 2013, the Pan American Health Organization (PAHO) issued an epidemiological alert due to the detection of the first local cases of CHIK in the Americas [12]. Since that time, this arboviral disease has arrived in the region of the Americas to stay. In some Latin American (LA) and Caribbean countries, CHIK has become a major public health problem. During 2014, transmission was rapidly established in places where dengue was present [13]. However, the 2014 outbreak was only the beginning of a new endemic disease, with some LA areas demonstrating stabilization of its transmission with a decrease in its incidence, while in others, a significant increase during 2015 was observed $[14,15]$.

According to the special bulletin issued by the Dominican Republic Ministry of Public Health, CHIK was found for the first time in the Caribbean Islands in December 2013 and reached 264,444 cases by June 2014, with 46,115 new cases in the Dominican Republic, among which 4721 infections were confirmed by laboratory tests and the number of deaths held at 21 [16]. According to PAHO, by August 2015, autochthonous transmissions were detected in 33 countries and territories of the Americas $(27$ countries and territories in the Caribbean, three countries in Central America, one country and one territory in South America, and one country in North America). LA has reported nearly one million cases; only Colombia (total population, 45 million inhabitants) has issued a report of $>200,000$ cases during the first 4 months of 2015 [17].

According to the Centers for Disease Control and Prevention (CDC), a total of 4702 CHIKV cases were reported to ArboNET from US territories for 2014. By 2015, cases from 36 US states were reported. The majority of cases occurred in travelers returning from affected areas, but a total of 108 cases of autochthonous CHIKV have been reported from US territories. All reported cases that were locally transmitted were reported from Florida, Puerto Rico, and the US Virgin Islands [18].

At present, CHIK has become one of the infectious diseases of this millennium that have crossed several international geographic boundaries within a short time period. The factors probably associated with the spread of the virus include the following: increased air travel, the previous lack of exposure of human populations, the invasion of A. albopictus from its native Asia into Africa, and into southern Europe, facilitated by increased global commerce and a series of adaptive mutations of CHIKV strains that mediated enhanced virus transmission by A. albopictus [19]. CHIK symptoms usually begin 4-8 days after a mosquito bite, but can appear anywhere from 2 to 12 days. Abrupt onset of fever, often accompanied by joint pain and prostration, are the most common symptoms. Additional signs and symptoms include myalgia, headache, nausea, fatigue, conjunctivitis, pain behind the eyes, sore throat, and skin rash [20].
This vector-borne disease commonly exhibits a self-limited course of arthritis, usually lasting for a few days or that may be prolonged to weeks [20,21]. Joint pain is usually intense and constant or intermittent. Joint swelling is often symmetrical in distribution, involving predominantly the small finger joints, wrists, feet, and ankles, with significant soft tissue swelling, and synovitis and tenosynovitis, a clinical picture evocative of rheumatoid arthritis (RA) [22]. However, in 10-60\% of cases, joint pain may become chronic and persist for up to $3-5$ years $[20,23]$. Characteristic severe axial pain presents in $28-32 \%$ of subjects, which is responsible for the characteristic "bended walker" appearance, in reference to the posture that patients assume with the resulting musculoskeletal involvement.

Furthermore, American College of Rheumatology/ European League Against Rheumatism (ACR/EULAR) classification criteria for RA may be fulfilled: rheumatoid factor and anti-citrullinated peptide antibodies are positive in 25 $43 \%$ of cases $[24,25]$. Bouquillard and Combe reported on 21 specific cases of RA that developed after CHIK, suggesting that the alphavirus could be triggering the RA [25]. Additionally, Miner et al. [24] reported 10 North American travelers who contracted the virus while visiting Haiti as a group demonstrated similar clinical features and comparable immunological phenotypes between patients with CHIKV and RA (seronegative). This clinical and immunologic overlapping highlights a need for rheumatologists to consider CHIKV infection when evaluating patients with recent-onset, symmetric polyarthritis. Furthermore, post-CHIK chronic inflammatory rheumatism is one of the consequences that are currently impacting endemic countries. Recently, RodríguezMorales et al. performed modeled weighted estimations based on pooled data, which estimated that about 400,000 patients would develop post-CHIK chronic inflammatory rheumatism in the Americas [26].

Rosario et al. in the Dominican Republic reported a recent experience with CHIK in general population and also in a group of patients with RA undergoing biological therapies [27]. A total of 514 patients presented for the first time at the clinic with complaints of musculoskeletal pain, 473 (92\%) exhibiting symmetric polyarthralgias, $344(67 \%)$ arthritis, and $385(75 \%)$ skin rash. The great majority, 457 (89\%), exhibited good clinical response to non-steroidal anti-inflammatory drugs (NSAID), 370 (72\%) required lowdose steroids, and only five patients $(0.97 \%)$ needed methotrexate therapy. On the other hand, of a total of 328 patients with RA on biological treatment, 53 exhibited CHIK-related musculoskeletal manifestations, 51 (96\%), symmetrical polyarthralgias, $25(47 \%)$, arthritis, and $13(24 \%)$, tendon involvement. The majority of the $51(96 \%)$ patients responded to NSAID, among whom 23 patients responded only partially and, in total, 25 (47 \%) required low-dose steroids. The majority of patients responded to symptomatic therapy, and there was no need to alter ongoing treatment with 
synthetic or biological disease-modifying anti-rheumatic drugs (DMARD).

During the past XIX Pan American League of Associations for Rheumatology (PANLAR) 2016 Congress held in Panama, five clinical studies associated with clinical manifestations, diagnostic imaging, and disease biomarkers related with CHIK fever were presented. Saaibi et al. evaluated joint enthesis and soft tissue structures by ultrasound (US) in 68 cases and found that CHIK fever exhibited more inflammation by US than suggested by clinical examination (abstract 3,274,137). Hernández et al., in a population of Villahermosa-Mexico, described the clinical and biochemical characteristics of the affected population at a primary-care service. Specific clinical features included distal symmetric polyarthritis and neck pain. Hunched posture $(100 \%)$, acral edema $(72.6 \%)$, and posterior cervical lymphadenopathy $(64 \%)$ were the most common and characteristic clinical signs (abstract $3,275,105)$. Sepúlveda et al., in a tertiary-care center in Chiapas-Mexico, studied clinical profiles, inflammatory biomarkers, disability, and chronicity in patients with CHIK. These authors found that acute symptoms of CHIK fever are frequent and produced high rates of disability; near one half of patients developed sub-acute and chronic arthritis. Inflammatory biomarkers, disability, and (DAS-28) remained high when compared with cases in which the infection resolved (abstract 3,287,762). Losanto et al. in Paraguay described the clinical characteristics of a population treated at a secondary-care hospital, highlighting the high frequency of hand and foot involvement, leading to several degrees of disability and impairments, and even to work absenteeism, due to the intensity of the pain. Although the follow-up period was short, excellent response to treatment with corticosteroids was highlighted (abstract $3,287,765$ ). Caballero et al., in a population of Barranquilla-Colombia, found that the acute clinical picture of CHIKV infection in patients with rheumatic disorders included fever, arthralgia/arthritis, and skin rash. In addition, sub-acute/chronic phases of CHIKV infection in patients with rheumatic disorders were characterized by small-joint involvement in a symmetric pattern. The authors' data suggested that immunosuppressive treatment did not affect the clinical manifestations of CHIKV infection (abstract 3,287,793) [28].

Recent publications have challenged the conventional view of the benign nature of CHIKV infection [29, 30]. The severe form of CHIKV infection may be associated with multiple organ failure, hepatitis, meningitis, nephritis, encephalitis, bullous dermatitis, myocarditis, and cardiac arrhythmias. While severe or atypical manifestations of CHIKV infection are rare, the overall mortality rate of these complications is high: nearly $10 \%$ [31]. The underlying pathophysiology of some of these complications is not yet fully understood [32]. Some studies suggested an association between severe manifestations and intense cytokine response with elevated levels of TNF, IL6, and IL13, or some specific genomic sequences of CHIKV [32].

Human-caused environmental changes, such as climate change, the globalization of international exchange, and disordered urban growth, are some factors that aid in the emergence and dissemination of human infectious diseases transmitted by vectors [2, 33-35]. The emergence of yellow fever, dengue, CHIK, and Zika represents a story of human behavior driving vector adaptation that, in turn, has driven viral adaptation [34]. Outbreaks of CHIK comprise a challenge for health care systems because of the high attack rate on the population. The economic burden in terms of the out-of-pocket (OOP) health expenditures incurred by an affected population was reported [35]. The major share $(47 \%)$ of the costs was utilized for purchasing medicines, but transportation costs (17\%), consultations (16\%), and diagnoses (9\%) also contributed significantly to the total OOP health expenditure. OOP was more than double the patients' average monthly family incomes. Governments should attempt to ensure comprehensive financial protection by covering the costs of health care, along with those involving loss of productivity, especially in developing LA countries, where the budget is designed to fight poverty, lack of education, the epidemiological and demographical transition, and attention to other health priorities [36, 37].

Best protection to date is prevention, in that there is no specific treatment for CHIK and vaccines are in developmental phases [33]. The resurgence of epidemics caused by arthropod-borne viruses (arboviruses), such as dengue, Ross River virus, West Nile virus, Zika, and CHIK, has intensified the importance of effective communication by health authorities for prevention and control. Health communication and risk communication have evolved from vertical processbased strategies based on propaganda and fumigation campaigns, to action for behavioral and belief change, in order to focus on community involvement and public approaches. There continues to be limited research evaluating the impact of these strategies, especially in the case of CHIK, which can be explained by the constant confusion among arboviral diseases. Furthermore, although it has been proven globally that one of the models recommended in the case of dengue is COMBI (communication for behavioral impact) and that the prevention and control of both viruses is similar, interventions for CHIK continue to be based on IEC (information, education, and communication) [38].

National health care systems should conduct epidemiological and entomological investigations, as well as establishing vector control measures; provide effective interventions for primary-, secondary-, and tertiary-prevention; formulate evidence-based outbreak management plans with separate 
consideration for low-, middle-, and high-income countries; provide technical support and guidance for the effective management of cases and outbreaks; and improve their reporting systems, in addition to developing and maintaining the clinical and laboratory capacity to detect and confirm cases, educate health providers, manage patients, and implement social communication strategies in order to engage the community in reducing the presence of the mosquito vectors [39]. However, it is noteworthy that, despite current vector control measures, A. aegypti and A. albopictus have maintained stable or even growing populations from rural to urban settlements and from lower to higher elevations. In addition to the lack of garbage collection, poor municipal piped-water supplies, uncontrolled urban development, and insecticide resistance, the weaknesses of vector control methodologies have undoubtedly contributed to maintaining abundant $A$. aegypti and A. albopictus populations in LA [40, 41].

The World Health Organization (WHO) reported in May 2016 allows us to determine that there have been an increasing number of autochthonous CHIK cases, which determines the probability of resistance and mutations [42] and which could lead to future new epidemics with bizarre manifestations of the same virus.

CHIK can no longer be considered a purely benign, selflimited, rare, and exotic diseases limited to the hotter parts of Africa, Asia, and Oceania. Currently, this arboviral disease is considered a global major public health challenge and economic threat. Are our national health care systems and rheumatologists prepared for CHIK?

Contributors CP, RM-L, CV-C, and DV contributed equally to the paper, wrote the manuscript together, and approved the final version.

\section{Compliance with ethical standards}

Disclosures None.

\section{References}

1. Fauci AS, Morens DM (2012) The perpetual challenge of infectious diseases. N Eng1 J Med 366(5):454-461. doi:10.1056 /NEJMra1108296

2. Lima-Camara TN (2016) Emerging arboviruses and public health challenges in Brazil. Rev Saude Publica 50. doi: 10.1590/S15188787.2016050006791

3. Mahendradas P, Avadhani K, Shetty R (2013) Chikungunya and the eye: a review. J Ophthalmic Inflam Infect 3(1):35. doi:10.1186/18695760-3-35

4. Thiberville SD, Moyen N, Dupuis-Maguiraga L, Nougairede A, Gould EA, Roques P, de Lamballerie X (2013) Chikungunya fever: epidemiology, clinical syndrome, pathogenesis and therapy. Antivir Res 99(3):345-370. doi:10.1016/j.antiviral.2013.06.009

5. Robillard PY, Boumahni B, Gérardin P, et al. (2006) Vertical maternal fetal transmission of the Chikungunya virus. Ten cases among 84 pregnant women. Presse Med 35:785-788
6. Robinson MC (1955) An epidemic of virus disease in Southern Province, Tanganyika territory, in 1952-53. I. Clinical features. Trans R Soc Trop Med Hyg 49(1):28-32

7. Lumsden WH (1955) An epidemic of virus disease in Southern Province, Tanganyika territory, in 1952-53. II. General description and epidemiology. Trans R Soc Trop Med Hyg 49(1):33-57

8. Weaver SC, Forrester NL (2015) Chikungunya: evolutionary history and recent epidemic spread. Antivir Res 120:32-39. doi:10.1016/j.antiviral.2015.04.016

9. Staples JE, Breiman RF, Powers AM (2009) Chikungunya fever: an epidemiological review of a re-emerging infectious disease. Clin Infect Dis 49(6):942-948. doi:10.1086/605496

10. Simon F, Parola P, Grandadam M, et al. (2007) Chikungunya infection: an emerging rheumatism among travelers returned from Indian Ocean islands. Report of 47 cases. Medicine 86(3):123137. doi:10.1097/MD/0b013e31806010a5

11. World Health Organization. Chikungunya (2016) Fact sheet $\mathrm{N}^{\circ} 327$. Updated (April 2016) http:/www.who.int/mediacentre/factsheets/fs327/en/. (Accessed Jul 2016

12. Organización Panamericana de la Salud/ Organización Mundial de la Salud (2016) Alerta Epidemiológica. Fiebre por Chikungunya. 9 de diciembre del 2013. Available at: http://www.paho.org/hq/index. php?option=com_docman\&task=doc_view\&gid=23807\&Itemid (Accessed Jul 2016)

13. Alfaro-Toloza P, Clouet-Huerta DE, Rodríguez-Morales AJ (2015) Chikungunya, the emerging migratory rheumatism. Lancet Infect Dis 15(5):510-512. doi:10.1016/S1473-3099(15)70160-X

14. Jiménez-Canizales CE, Medina-Gaitán DA, Mondragón-Cardona ÁE, Rodríguez-Morales AJ (2015) From imported to an endemic disease: impact of Chikungunya virus disease in the hospital epidemiology, Tolima, Colombia, 2014-2015. Recent Pat Antiinfect Drug Discov 10(1):64-66

15. Rodríguez-Morales AJ, Bedoya-Árias JE, Ramírez-Jaramillo V, Montoya-Árias CP, Guerrero-Matituy EA, Cárdenas-Giraldo EV (2015) Mapping chikungunya fever in municipalities of one coastal Department of Colombia (Sucre) using geographic information system (GIS) during 2014 outbreak: implications for travel advice. Travel Med Infect Dis 13(3):256-258. doi:10.1016/j. tmaid.2015.05.007

16. Boletín especial (2014) MISP \# 13. 20-08. http://www.paho. org/dor/images/stories/archivos/chikungunya/boletin_chikv_no3_2014_6_09.pdf (Accessed Jun 2016)

17. Boletín Epidemiológico Semanal (Semana epidemiológica número 17 de 2015). Available at: http://www.ins.gov.co/boletinepidemiologico/Boletn\%20Epidemiolgico/2015\%20Boletin\%20 epidemiologico\%20semana\%2017.pdf (Accessed Aug 2015)

18. Centers for Disease Control and Prevention (2015) Chikungunya virus http://www.cdc.gov/chikungunya/geo/united-states.html (Accessed Aug 2015

19. Weaver SC, Lecuit M (2015) Chikungunya virus and the global spread of a mosquito-borne disease. N Engl J Med 372(13):12311239. doi:10.1056/NEJMra1406035

20. Ali Ou Alla S, Combre B (2011) Arthritis after infection with Chikungunya virus. Best Pract Res Clin Rheumatol 25:337-346. doi:10.1016/j.berh.2011.03.005

21. Win MK, Chow A, Dimatatac F (2010) Chikungunya fever in Singapore: acute clinical and laboratory features, and factors associated with persistent arthralgia. J Clin Virol 49(2):111-114. doi:10.1016/j.jcv.2010.07.004

22. Ganu MA, Ganu AS (2011) Post-Chikungunya chronic arthritisour experience with DMARDs over two year follow up. J Assoc Physicians India 59:83-86

23. Schilte C, Staikowsky F, Couderc T, Madec Y, Carpentier F, Kassab S, Albert ML, Lecuit M, Michault A (2013) Chikungunya virusassociated long-term arthralgia: a 36-month prospective longitudinal 
study. PLoS Negl Trop Dis 7(3):e2137. doi:10.1371/journal. pntd.0002137

24. Miner JJ, Aw-Yeang HX, Fox JM, et al. (2015) Chikungunya viral arthritis in the United States: a mimic of seronegative rheumatoid arthritis. Arthritis Rheumatol 67(5):1214-1220. doi:10.1002 /art.39027

25. Bouquillard E, Combe B (2009) Rheumatoid arthritis after Chikungunya fever: a prospective follow-up study of 21 cases. Ann Rheum Dis 68(9):1505-1506. doi:10.1136/ard.2008.097626

26. Rodriguez-Morales AJ, Cardona-Ospina JA, Villamil-Gómez W, Paniz-Mondolfi AE (2015) How many patients with postChikungunya chronic inflammatory rheumatism can we expect in the new endemic areas of Latin America? Rheumatol Int 35(12): 2091-2094. doi:10.1007/s00296-015-3302-5

27. Rosario V, Muñoz-Louis R, Valdéz T, et al. (2015) Chikungunya infection in the general population and in the patient with rheumatoid arthritis on biological therapy. Clin Rheumatol 34(7):12851287. doi:10.1007/s10067-015-2979-x

28. Abstracts PANLAR (2016) J Clin Rheumatol 3(22):108-163. doi:10.1097/RHU.0000000000000372

29. De la Hoz JM, Bayona B, Viloria S, et al. (2015) Fatal cases of Chikungunya virus infection in Colombia: diagnostic and treatment challenges. J Clin Virol 69:27-29. doi:10.1016/j.jcv.2015.05.021

30. Economopoulou A, Domínguez M, Helynck B, et al. (2009) Atypical Chikungunya virus infections: clinical manifestations, mortality and risk factors for severe disease during the 20052006 outbreak in Réunion. Epidemiol Infect 137(4):534-541. doi:10.1017/S0950268808001167

31. Couderc T, Lecuit M (2015) Chikungunya virus pathogenesis: from bedside to bench. Antivir Res 121:120-131. doi:10.1016/j. antiviral.2015.07.002

32. Lohachanakul J, Phuklia W, Thannagith M, et al (2012) High concentrations of circulating interleukin-6 and monocyte chemotactic protein- 1 with low concentrations of interleukin- 8 were associated with severe Chikungunya fever during the 2009-2010 outbreak in Thailand. Microbiol Immunol 134-138. doi: 10.1111/j.13480421.2011.00417.x
33. Rolph MS, Foo SS, Mahalingam S (2015) Emergent chikungunya virus and arthritis in the Americas. Lancet Infect Dis 15(9):10071008. doi:10.1016/S1473-3099(15)00231-5

34. Morens DM, Fauci AS (2014) Chikungunya at the door Déjà vu all over again? N Engl J Med 371(10):885-887. doi:10.1056/NEJMp1408509

35. Vijayakumar K, George B, Anish TS, et al. (2014) Economic impact of chikungunya epidemic: out-of-pocket health expenditures during the 2007 outbreak in Kerala, India. Southeast Asian J Trop Med Public Health 44(1):54-61

36. Pineda C, Caballero-Uribe CV (2015) Challenges and opportunities for diagnosis and treatment of rheumatoid arthritis in Latin America. Clin Rheumatol 34(Supp1 1):S5-S7. doi:10.1007/s10067-015-3019-6

37. Cardona-Ospina JA, Vera-Polania F, Rodriguez-Morales AJ (2016) Chikungunya or not, differential diagnosis and the importance of laboratory confirmation for clinical and epidemiological research: comment on the article by Rosario et al. Clin Rheumatol 35(3):829 830. doi:10.1007/s10067-015-2995-x

38. WHO. Guidelines for Prevention and Control of Chikungunya Fever 2009. http://www.wpro.who.int/mvp/topics/ntd/Chikungunya WHO_SEARO.pdf (Accessed July 2016)

39. European Center for Disease Prevention and Control. Chikungunya. http://ecdc.europa.eu/en/healthtopics/chikungunya fever/Pages/index.aspx (Accessed July 2016)

40. Fernández-Salas I, Danis-Lozano R, Casas-Martínez M, et al. (2015) Historical inability to control Aedes aegypti as main contributor of fast dispersal of chikungunya outbreaks in Latin America. Antivir Res 124:30-42. doi:10.1016/j.antiviral.2015.10.015

41. Vijayan V, Sukumaran S (2016) Chikungunya virus disease: an emerging challenge for the rheumatologist. J Clin Rheumatol 22(4):203-211. doi:10.1097/RHU.0000000000000396

42. American Health Organization/World Health Organization. Number of cumulative cases 2015. Available at: http://www.paho. org/hq/index.php?option $=$ com_docman\&task=doc view\&Itemid=270\&gid=33091\&lang=en 